\title{
Improvements in modern weapons systems: the use of dielectric materials for the development of advanced models of electric weapons powered by brushless homopolar generator
}

\section{Daniela Georgiana GOLEA}

PhD student, "Mihai Viteazul" National Intelligence Academy, Bucharest, Romania kolerdaniela@yahoo.com

\section{Lucian Ștefan COZMA}

PhD in Military Sciences, National Defense University "Carol I", Bucharest, Romania PhD student, Faculty of Physics, Magurele, University of Bucharest, Romania lucian.stefan@yahoo.fr

\begin{abstract}
Although the idea of making electric weapons has emerged since the beginning of the 20th Century, the great number of technological problems made that such technology was not developed. During the Cold War, the US strategic programme Space Defensive Initiative paid a special attention to this category of weapons, but the experiments have demonstrated that the prototypes are too big, very heavy, and involve a very high energy consumption and low reliability. Under these circumstances, the authors have noticed the trend to design new types of electric weapons starting from the hybridization of some technologies: the compressed flux weapons, the plasma electromagnetic cannon, the electromagnetic weapons as the coil-gun and rail-gun.
\end{abstract}

Keywords: electric weapons, rail gun, electromagnetic ammunition, hybrid technologies, plasma detonator.

\section{Introduction}

Since the beginning of the $X^{\text {th }}$ Century there have been concerns about the development of the electric weapons capable of equating and even exceeding the performance of firearms by using the electromagnetic forces. In order to see how this technology evolved and what prospects it opens, we will continue by giving five examples: the early electric weapons proposed by the inventor Fauchon Vileplee ${ }^{1}$; the electric weapon model analyzed by Ladislav Zenise ${ }^{2}$; the model experienced by the French ${ }^{3}$ in the late 90s, and the model recently analyzed by lt.col. Laurian Gherman ${ }^{4}$ and the improvements proposed by the authors of this paper. In the opinion of the authors, the achievement of a performing electric weapon implies not only the use of a suitable power supply, but also a munition capable of generating its own strong magnetic field. The experiments with passive electromagnetic ammunition have shown that the ratio electric power-to-ballistic performances is unsatisfactory.

\section{History of the concept and its possibilities for development}

Inventory and experimentation activities at the beginning of the XXth Century did not manage to overcome the many technological impediments, so that until the outbreak of the Second World War a realistic model of electric weapon had not been developed.

\footnotetext{
${ }^{1}$ Andre Louis Octave Fauchon-Villiplee (1869-1959) french inventor, even during the First World War he invented and improved various models of electric guns, projectiles or power systems for these electric guns.

${ }^{2}$ Ladislav Zenisek, Mașini electrice speciale, Editura Tehnică, București 1959, pp. 83-87, Apud A. Sternfeld, Lety do vesmiru, Praga, 1956.

${ }^{3}$ The model experimented by the specialists of GEC-Alsthom in 1997.

${ }^{4}$ Laurian Gherman, Two fluxes multistage induction coilgun, articol în Review of the Air Force Academy, no. 3

(30), Brașov, 2019.
} 

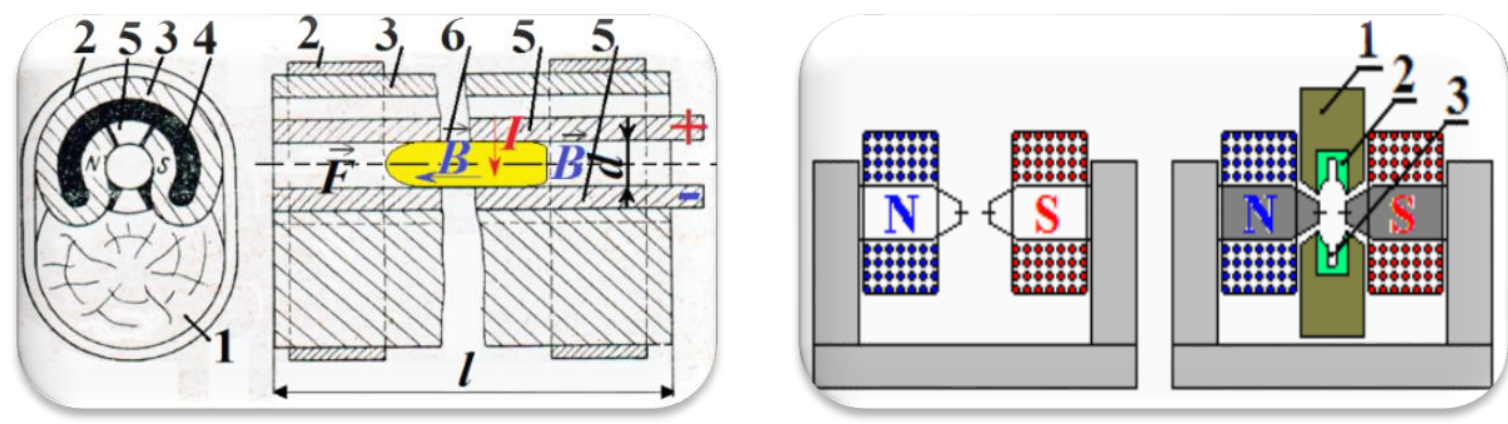

Fig. 1 The electric weapon (left) scheme presented in 1959 by Ladislav Zenisek (explanations in the text); the general model is that of a hybrid electric weapon, the mixed version of the rail-gun and the coil-gun. In the Semisek model the rail-gun principle is applied, the current being induced by two longitudinal metal bars, but also the coilgun principle, the traveling magnetic field being generated by the circular (electro)magnets that actually form the body of the gun barrel. The general model of a Weiss magnetic field concentrator (right), an electromagnet with a large induction, used in a hybrid rail-gun/coil-gun scheme: 1- insulating and reinforcement material, 2- highcurrent conductor rails, 3-profile for the projectile's reinforcement, through which it enters inside the rails. This scheme would allow an increase of the induction value to 10 Tesla, which would allow the required value of the current to be reduced in the rails;

Since the period of the First World War, the french inventor Fauchon Villeplee proposed the creation of some electric guns according his patents: FR 1,204,301 for an homopolar dynamo, FR 1,204,302 for an electric catapult, FR 21,159 for an electric gun, FR 22,432 for a new projectile type and launch method, FR 22,448 for an electric cannon, FR 29,135 for another type of electric cannon, FR 30,393 for an improved electric battery, FR 30,395 for an electric gun, FR 36,699 for an homopolar dynamo, FR 37,717 for another model of homopolar dynamo, FR 493,418 for an electric gun, FR 496,325 for improvements to the electric cannon, FR 505,134 for a new type of dynamo, FR 505,696 for an electric gun, FR506280 for o fuse of electric weapon projectile, FR 506,288 another type of electric gun, FR 506,289 for an accelerator and electric generator, FR 511,944 granted for an electric cannon, FR 655,931 for another type of electric cannon, FR 656,584 granted for homopolar generator, FR 695,639 for an collector circuit, FR 992,846 for an electric cannon, FR 992,847 granted for an rocket projectile, etc. Also, Kristian Olaf Birkeland (1867-1917) was an inventor who designed and built several types of electromagnetic weapons in the early XXth Century, when he made the first coil-gun.

In general, $F$. Villeplee has failed to obtain a compact model of his electric weapons, mainly because of the rudimentary technology of the power sources and the capacitors used, which were excessively bulky and generally poor. In fact, the same kind of problems faced the specialists much later, after the end of the Second World War. Even the use of Bitter-type bobbins or Weiss magnetic field concentrators did not manage to remove the major impediments that led to the impossibility of establishing some practical weapon models.

Over time, the simplistic model of the coil-gun electromagnetic accelerator was abandoned in favor of the rail-gun accelerator, but this model also encountered major problems caused by the mechanical interactions between the projectile and the magnetic rails. Regarding all these issues, the authors observed that the trend of technology development leads to the adoption of the following solutions: 

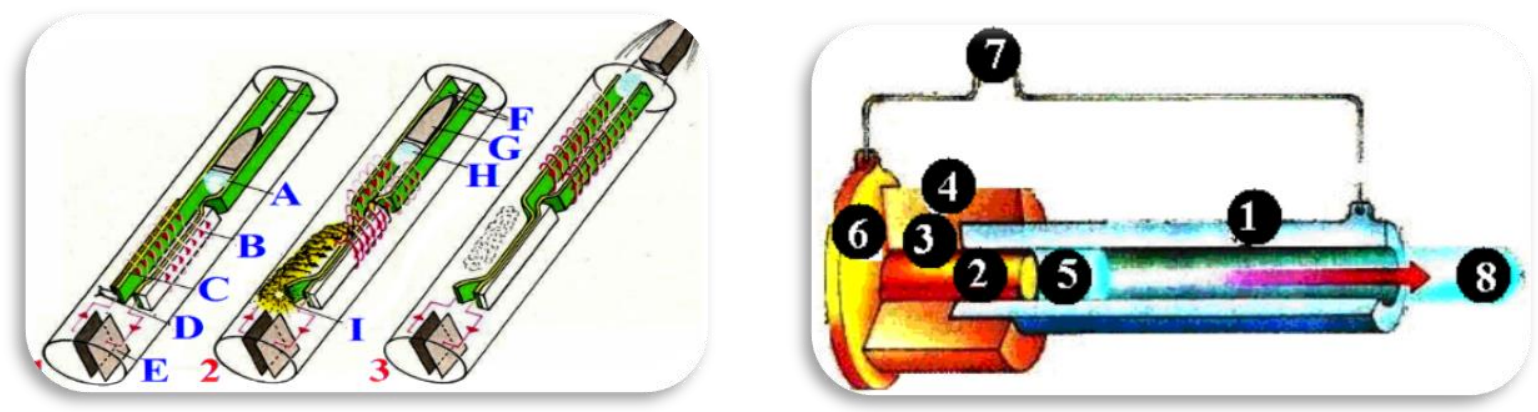

Fig. 2 On the left, the classic plasma electromagnetic cannon ${ }^{5}$ which could be a model for today electromagnetic ammo for railguns: 1 - the current is released from the capacitor in the metal rails, thus creating a very intense magnetic field; at the same time, the detonator produces the explosion by compressing the rails and thus amplifying the magnetic field intensity; this magnetic field very strongly rejects the plasma created in the space between the rails, the magnetic force being so high that it accelerates the projectile up to $10 \mathrm{~km} / \mathrm{sec}$ after a stroke of only 1 meter length. The system components: A- projectile shield; B- the magnetic field force lines; C- explosive; Ddetonator; E- capacitor; F- metallic rails; G- projectile; H- plasma; I- blasting with compression effect. On the right, the experimental device made by N. Graneau 6 , called "Water Arc Gun" and composed of a steel cylinder (1) $12 \mathrm{~mm}$ in diameter and a height of $100 \mathrm{~mm}$; at the lower side of this iron pipe is an cylindrical element (2) made of copper and insulated with respect to the element (1) and also externally by means of nylon insulating shrouds (3) and epoxy resin (4). This copper cylindrical element (2) serves as an electrode being solidar with a copper plate (6) which also represents the support element of the entire experimental device. This base plate is connected to the capacitor (7). At the same time, the cylindrical electrode (2) is also the bottom piece under the water column (5). By means of the capacitor whose terminals are connected to (6) and (1) respectively, a current of 60,000 Amps is discharged within the circuit. This produces a shock wave due to the internal repellent forces and the water is ejected through the tube (8) in the form of hot plasma but with such force that it can pierce an aluminum wall with a thickness of $6 \mathrm{~mm}$. The plasma jet speed is about $1 \mathrm{Km} / \mathrm{sec}$, about three times the speed of the sound. For aerospace applications and in order to improve the performance of the device, it is advisable to use a not the water, but "cold plasma", such fluid having a very good value of the electron mean free pass, in this way it can be obtained ejection speeds in the order of 10,000 Km/sec. Such "plasma cannons" have also been tested under the US "Star Wars" (SDI) programe. Under certain conditions, with such an electric cannon, we can obtain high-pressure steam necessary for a sonic weapon system.

* the use of active-passive electromagnetic ammunition, composed of an self-detonating cartridge (with self-destruct at the time of firing) type $\mathrm{FCG}^{7}$ (flux compression generator) and a passive electromagnetic projectile; at the time of firing, the explosion of the cartridge causes an intense magnetic field ${ }^{8}$ behind and around the projectile to interact with the magnetic field applied externally by means of the rail-gun;

* the use of the active electromagnetic ammunition based on the application of piezoelectric effect caused by the explosion of a small amount of powder ${ }^{9}$ which produces a mechanical compression effect on some piezoelectric elements; the piezoelectric elements discharge a high voltage pulse within a circuit composed of a capacitor and resistance, both located inside the projectile; this circuit will ultimately supply the external winding of the projectile; the described processes occur in very short time intervals, during the projectile is crossing the barrel (inside the coil-gun or rail-

\footnotetext{
${ }^{5}$ According $S \& V$ no. 760, Jan. 1981.

${ }^{6}$ According $S \& V$ no. 879, Dec. 1990.

${ }^{7}$ According the patent US 8723390 for Flux compression generator granted to Blaise L. Corbett, Jack L. Price, Albert J. Corda, Yehoshua D. Agassi, Walter D.

${ }^{8}$ According the patents US 4753153 for Electromagnetic railgun with a non-explosive magnetic flux compression generator granted to Louis J. Jasper, US 5016600 for Methods of generating and controlling a magnetic field without using an external power supply specification granted to Mohamed Hilal, US 5835545 for Compact intense radiation system granted to Peter J. Turchi, etc.

${ }^{9}$ Idea inspired by the patent US 5024137 for Fuel assisted electromagnetic launcher granted to Jon M. Schroeder.
} 
gun) the interaction between the outer magnetic field and the projectile's own field, leading to its better acceleration.

We will start our analysis with the classic weapon model presented by L. Zenisek (figure 1) since the 50s. This was composed of: the gun stock $\mathbf{1}$ of which the barrel $\mathbf{3}$ is fastened by means of bracelets $\mathbf{2}$. But the barrel $\mathbf{3}$ is in fact a permanent magnet or an electromagnet. The barrel has virtually the diameter of the cylindrical gap between the magnetic poles. The electrical current passes through two profiled copper rods, marked with 5, and the insulating material $\mathbf{4}$ serves as a mechanical protection against the dynamic effects occurring at the current passage. The friction of the projectile $\mathbf{6}$ in the barrel will be neglected because it will be considered to be moving on the magnetic cushion (magnetic levitation conditions).

From the relationship with which is calculated the force acting on a conductor of length $\boldsymbol{d}$ in which passes a current $\boldsymbol{I}$, into an magnetic field with induction $\boldsymbol{B}$, we obtain

$$
F=\vec{B} \cdot d \cdot I
$$

and the current is then calculated

$$
I=\frac{F}{\vec{B} \cdot d},
$$

in relation (1) we replace

$F=m \cdot a$,

then, from the uniformly accelerated motion equation (where $l$ is the length of the barrel) we obtain

$$
l=\frac{a}{2} t^{2}
$$

and results the necessary acceleration

$$
a=\frac{2 l}{t^{2}}
$$

from mathematical expression of initial speed of the projectile (muzzle velocity)

$v_{0}=a \cdot t$,

the time required for completion of the barrel results as the

$$
t=\frac{v_{0}}{a} \text {, }
$$

and if we replace $t$ in relation (5) we obtain the acceleration

$a=\frac{2 l}{t^{2}}=\frac{2 \cdot l \cdot a^{2}}{v_{0}^{2}}=\frac{v_{0}^{2}}{2 \cdot l}$,

by substituting the acceleration $a$ from relation (8) in relation (3) results

$$
F=m \cdot a=\frac{m v_{0}^{2}}{2 \cdot l}
$$

the time necessary for the projectile to pass the barrel is calculated from the relationship (8)

$$
t=\frac{2 \cdot l}{v^{2}}
$$

so that during the firing of the projectile, through the electric bars 5 must pass a total load of

$$
Q=I \cdot t=\frac{m v_{0}}{B \cdot d},
$$



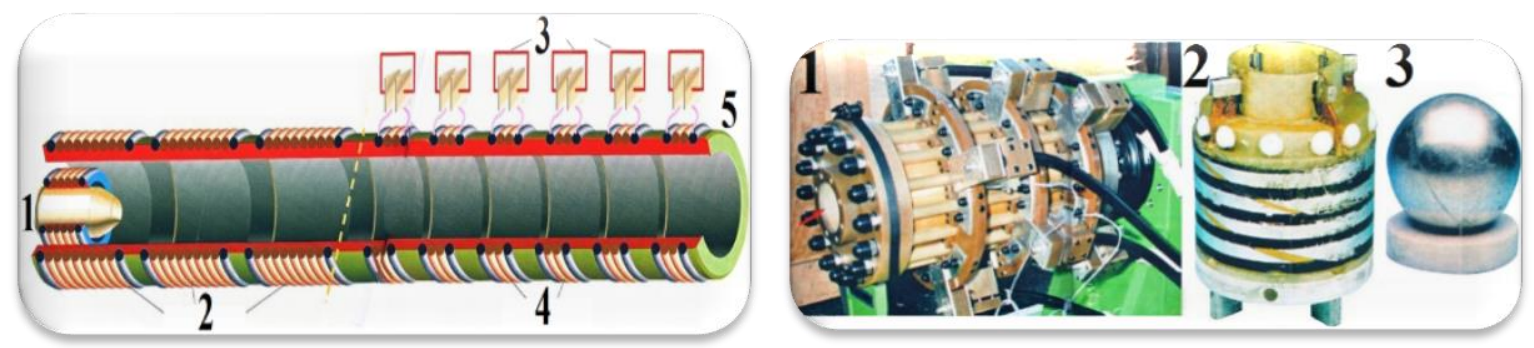

Fig. 3 A drawing depicting the electric weapon (coil-gun type) experimented by GEC-Alsthom ${ }^{10}$ : up-side, a drawing showing the main components of the electric cannon, 1- the projectile with its winding, 2- the starter coils, 3- the ultra-capacitor batteries, 4- the coils of the accelerator with traveling magnetic field ; 5- the muzzle. At the lower side: 1- the starting device consisting of three coils, 2- projectile with winding, 3- combat load. Our critique of this experimental weapon refers to the power supply, the winding and the projectile.

These mathematical relations stated by Zenisek were simply applied in a practical example: the mass of projectile $m=7,5$ grams $=7,5 \cdot 10^{-3} \mathrm{~kg}$, the projectile velocity $v_{0}=800 \mathrm{~m} / \mathrm{s}$, the induction $\vec{B}=0,5$ Tesla (5000 Gs), the diameter of the projectile $d=7,3 \mathrm{~mm}=7,5$ meters, the approximate barrel length $l=750 \mathrm{~mm}=0,75$ meters. The current required for acceleration must be:

$$
I=\frac{1}{2} m v^{2} \frac{1}{B \cdot \vec{d} \cdot l}=\frac{1}{2} \cdot 7,5 \cdot 10^{-3} \cdot 800^{2} \cdot \frac{1}{0,3 \cdot 7,5 \cdot 10^{-3} \cdot 0,75}=1,42 \mathrm{MA},
$$

and the passing of projectile through the barrel would last $t=\frac{2 l}{v_{0}}=\frac{2 \cdot 0,75}{800}=0,00187 \mathrm{sec} \approx 0,002 \mathrm{sec}$,

During this time, a total load of electric current would pass through the electric circuit

$Q=I \cdot t=1,42 \cdot 10^{6} \cdot 2 \cdot 10^{-3}=2,84 \cdot 10^{3} \mathrm{C}$,

for the projectile to get the kinetic energy

$W=\frac{1}{2} m v_{0}^{2}=\frac{1}{2} \cdot 7,5 \cdot 10^{-3} \cdot 800^{2}=2,4 \mathrm{~kJ}=245 \mathrm{kgf} \cdot \mathrm{m}$.

The same result can be reached from the relationship (1) in another way, since the relation (1) results:

$W=\vec{B} \cdot d \cdot l \cdot I=0,3 \cdot 7,5 \cdot 10^{-3} \cdot 0,75 \cdot 1,42 \cdot 10^{6}=2,4 k J$,

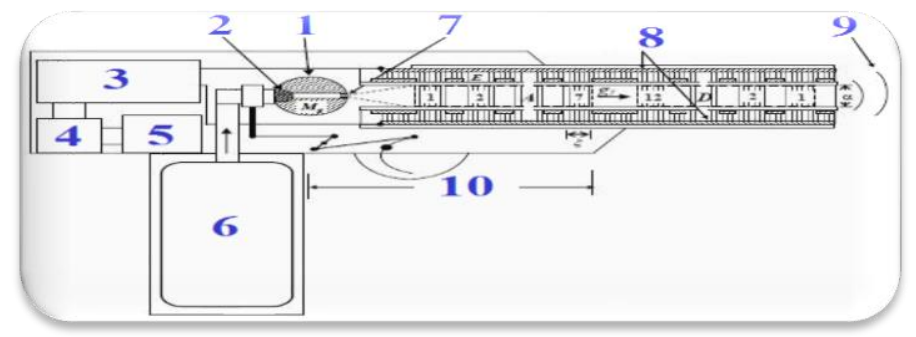

Fig. 4 An model of portable shock wave generator, according to patent PI0805046-5 of July the 31th, 2008. Such a weapon can produce detonations in which the shockwave has a velocity greater than $100 \mathrm{~km} / \mathrm{sec}$ and produces pressures greater than $1010 \mathrm{~N} / \mathrm{m}^{2}$, that shock wave may even destroy the armored plates. Such a weapon consists of: 1- tungsten carbide with a density of $15,8 \mathrm{~kg} / \mathrm{m}^{3}$ and a mass of $4,23 \mathrm{~kg}$, the radius of $40 \mathrm{~mm}$; 2- the filter; 3the high voltage transformer; 4- the ultra low frequency oscillator; 5- power supply; 6- an tank containing water; 7- the nozzle with $0,38 \mathrm{~mm}$ diameter; 8 - the capacitor plates; 9 - the spherical shock wave; 10 - the distance approx. $150 \mathrm{~mm}$.

\footnotetext{
${ }^{10}$ According $S \& V$ no. 954 , mars 1998, pp. 124-127.
} 
where the product $\vec{B} \mathrm{dl}=\phi$ represents the total magnetic flux $[\mathrm{Wb}]$ in the electric gun barrel, therefore, the last equation shows that the electric rifle gives to the projectile a kinetic energy $W=\phi \cdot I$,

As Zenisek well observed, the current $\boldsymbol{I}$ would not be constant as assumed in the calculation assumption, especially if we take into account the variation of impedance while the projectile passes through the barrel. This means that $I=f(t)$ and if the induction poles were formed by an electromagnet, the magnetic flux would be time-dependent $\phi=g(t)$, so that the equation (12) would be more complicated. Thus, it has neglected even the fact that it is a transitory phenomenon and not a stationary regime. Zenisek observed, however, that the weapon could be constructed so that the current $\boldsymbol{I}$ is also the excitation current of the magnet $\mathbf{3}$ (fig.1), so the gun is based on the same principle as the electric engines with series comutation. The electric bars 5 (fig.1), if executed in a massive construction, would probably resist to the thermal and dynamic effect for an short-circuit of 1,42 MA in 0,002 seconds. A difficult problem in Zenisek's vision was, however, the effect of the passage resistance between these bars 5 and the projectile 6 (fig.1), and if under the influence of heat (the Joule effect) the projectile would not be destroyed. Despite all these issues, Zenisek noticed that the biggest obstacle in making such a weapon is to solve the problem of the very rapid renewal of the electric load of $2,84 \mathrm{kC}=0,8 \mathrm{Ah}$.

He considered that at a load voltage of $220 \mathrm{~V}$ the capacitor should have a capacity of $12,8 \mathrm{~F}$ and such a capacitor, Zenisek appreciated in 1959, that would weigh about 100 tons $^{11}$. Another problem that dwarfed Zenisek almost 60 years ago was if this capacitor could be discharged within 0,002 seconds, and that the exponential flow of the discharge current would mean an uneven acceleration of the projectile.

In spite of these problems, Zenisek noted optimistically that the electric load of 2,84 kC $=0,8 \mathrm{Ah}$ can be theoretically accumulated in just 8 grams of active mass of a lead-acid accumulator, and ensure the power supply of an electric weapon, theoretically, only an electric battery of $10 \sim 12$ grams would be needed, in the form of a cartridge. This battery should have a very large surface so that it can be discharged through a short circuit in a timeframe of two thousandths of a second. Of course, he could not know that in the next few decades the capacitor technology would evolve so much that obtaining a capacity of 12,8 Farad would be almost a bagatelle ${ }^{12}$. At present, the development of graphene capacitor technology is being attempted.

This is a nano-structure consisting of a single layer of graphite with atoms arranged in hexagonal meshes, these "foils" being characterized by a very good electrical conductivity. As the ratio between the surface and the weight of the graphene is nearly $2700 \mathrm{~m}^{2} / \mathrm{gram}$, it results in a capacity of about $500 \sim 600 \mathrm{~F} / \mathrm{gram}$. Therefore, the energy-to-mass ratio is above $85 \mathrm{Wh} / \mathrm{kg}$ ie more than $300 \mathrm{~kJ} / \mathrm{kg}$; and if should be used carbon nanotubes with diameters between 1 and $3 \mathrm{~nm}$, with the length between 20 and $100 \mu \mathrm{m}$, the surface-to-mass ratio may exceed $3000 \mathrm{~m}^{2}$ /gram. On the other hand, in the case of Zenisek, the magnetic induction was limited to the value of 0,5 Tesla, much below the technological possibilities of this moment. At the same time, obtaining this induction can be done with the help of small Weiss electromagnets, as shown in Fig. 1. If the Bitter Coil ${ }^{13}$ is used for electric gun windings, a very high magnetic induction value can be obtained.

\footnotetext{
${ }^{11}$ Of course, the performances of the capacitors has greatly evolved so that, for example, a nanotechnology-based capacitor may have the capability of thousands of farad despite some small or very small dimensions (and weight). ${ }^{12}$ See the patent US 4,870,538 granted in 1989 to Ewart Baldwin for a high energy capacitor.

${ }^{13}$ The Bitter Coil or Bitter electromagnet or Bitter solenoid is a type of disc electromagnet currently used in some scientific research in order to create extremely strong magnetic fields (up to 45 Tesla).
} 
Working in conditions far superior to those of the late 50s, the French specialists from GEC-Alsthom ${ }^{14}$ experienced in 1997 a small electric cannon of $120 \mathrm{~mm}$ caliber, capable of accelerating projectiles weighing several kilograms, to a velocity in the order of miles per second. Thus, according to the published data ${ }^{15}$, the GEC-Alsthom electric cannon was made of three starting coils (which accelerated the projectile up to $300 \mathrm{~m} / \mathrm{sec}$ ) each powered by a capacitor, the total energy being 2,2 MJ. The second phase of accelerating the projectile corresponded to the action of a traveling magnetic field, using 33 coils powered through a succession of very fast sequences by 33 capacitors totaling 6,3 MJ of energy. In this way, projectiles with a mass of $4,3 \mathrm{~kg}$ could be launched at speeds of the order of $800 \mathrm{~m} / \mathrm{sec}$ or projectiles of $1 \mathrm{~kg}$ at the speed of $2400 \mathrm{~m} / \mathrm{sec}$. Encouraged by these results, the French estimated at the beginning of 1997 that they would be able to accelerate $5 \mathrm{~kg}$ massive projectiles at 2500 $\mathrm{m} / \mathrm{sec}$ muzzle velocity, with an effective range of 5000 meters.

The French used coils with length (measured along the longitudinal axis) of 0,14 meters, in which strong currents were discharged in 250 millisecond intervals, resulting in 60 Tesla inductions. At that time it was appreciated that the setting up of some coils capable of withstanding $400 \mathrm{kA}$ discharges at $20 \mathrm{kV}$ voltage could allow the obtaining of 100 Tesla inductions. Undoubtedly, a special improvement of these types of weapons could be represented by the use of $F C G^{16}$ technology in the functional scheme of the ammunition used by a railgun... The FCG and EMP-bomb technologies can be used both in the construction of the cartridge and projectile and also to create certain effects on the target, as a combat load.

Lt. col. Laurian Gherman has more recently ${ }^{17}$ presented an electric weapon model with double-flow, multi-stage coil, in which the multi-stage accelerator was made up of a set of three elements with different functions: the induction coil, the coil designed to create the magnetic field and the annular element (projectile). According to published data, in the computerized simulation at the first acceleration stage, the potential was $3600 \mathrm{~V}$ and the time interval of 1,7 $\mathrm{ms}$; in the second stage, $5600 \mathrm{~V}$ in $1 \mathrm{~ms}$; in the third stage $9600 \mathrm{~V}$ during $0,55 \mathrm{~ms}$ and fourth stage respectively, $11600 \mathrm{~V}$ in $0,4 \mathrm{~ms}$.

According to our research, the further development of the electric weapon schemes would fall into the following coordinates:

\# the improving of the bipolar, non-sulfatable, Lead-Acid electrochemical sources, with granular eletrodes and gelatinized electrolyte (compact secondary electrochemical sources having a good energy-to-mass ratio);

the improvement and compaction of brushless homopolar generators with dielectric material discs, according to U.S. Patent No. 5,051,905 granted to Richard Clark on April 18,2000 ;

the use of some compact models of the brushless DC engine according to the patent document ${ }^{18}$ entitled "Method and system for the realization of magnetic energy conversion".

The above solutions offer:

an electric motor with much better performances than all the types of electric motors made so far,

\footnotetext{
${ }^{14}$ Alsthom is a French industrial group based in Paris, whose business is in the field of electricity production and transport.

${ }^{15}$ S\&V no. 954, mars 1998, pp. 124-127.

${ }^{16}$ Flux compression generators (FCG); a pyrotechnic charge is disposed inside the core of a coil traversed by electric current; an electromagnetic pulse of high power is generated that destroys the electrical and electronic equipment but can also kill the combat personnel if the bomb explodes near people.

${ }^{17}$ Laurian Gherman, 'Two fluxes multistage induction coilgun", in Review of the Air Force Academy, nr. 3 (30), Brașov, 2019.

${ }^{18}$ Regular national deposit A2016 00540, OSIM-Bucharest.
} 
a brushless homopolar generator that uses dielectric disks instead of conductive material,

the use of Bitter $^{19}$ and Weiss ${ }^{20}$ coils for magnetic accelerators;

the using of ultra-capacitors with graphene or nanotubes.

In one of the new projects proposed by the authors, the capacitors and discharge circuits are completely removed from the scheme, the operation not being in the transient mode or dependent on the discharge times. The strong current is obtained directly from the brushless homopolar mini-generator, powered by the brushless electric motor, this latter powered by an advanced electrochemical source.

The traveling magnetic field traverses the Bitter coils and interacts with a magnetic projectile that produces its own magnetic field by short-circuiting an internal mini-condenser when the fire is executed. Thus, the coil-projectile is not induced by the magnetic field applied by the outer circuit, but it is used the interaction between the magnetic fields, one applied from the outside of the projectile, the other being the very own magnetic field of the projectile. For the time being, only an ante-design has been carried out, being selected the technologies considered appropriated, but as the experimental results will be obtained, we shall disseminate these results of our scientific research.

As early as September 2001, the american magazine Popular Mechanics published an interesting article on electromagnetic pulse weapons. That article specifically referred -among others- to the so-called compression flux bombs, which have been designed and built since the 1950s. This kind of electromagnetic pulse weapons is in fact a hybrid technology that partly uses the components of classical bombs but also elements specific to the electromagnetic weapons. The military science analyst Carlo Kopp has spoken on this subject in a report on electromagnetic weapons. Although the principle of operation and the principle schemes have long been published, the concrete technology of achieving and using these weapons in real combat situations remains little known.

Such an electromagnetic bomb is made up of a metallic cylinder and it has an external winding, the copper windings being called stator windings. The core of the cylindrical armature is filled with a high-explosive powder disposed within an shield against the induction current. The stator winding and the cylindrical armature are deflected through an empty gap. Also, inside the bomb is an electric source, which is coupled to a bank of capacitors. This source powered the stator winding.

The operation of such a bomb can be summarized as follows: a switch will connect the capacitors to the stator winding so that the winding will be swept by an intense current. A high induction magnetic field will be obtained. A classic fuse shall ignite the the pyrotechnic load, and the shock wave of its explosion goes very fast through the longitudinal axis of the armature. As this shock wave advances, the armature contacts the stator winding coil, which creates a strong short circuit. The rapid movement of the short-circuit wave produces the effect of compressing the magnetic field, generating a very high magnetic pulse. This electromagnetic shock wave can only affect a relatively small surface but however, it has the potential to produce significant destructive effects.

In our study, we try to see how this operating principle can be applied to portable electromagnetic weapons. In order to develop new rail-gun ${ }^{21}$ weapons, we have in mind to

\footnotetext{
${ }^{19}$ Bitter Coil was invented in 1933 by the American physicist Francis Bitter (1902-1967) and used to obtain magnetic fields with inductions ranged between 20 and 45 Tesla.

${ }^{20}$ Special electromagnet used as magnetic field concentrator; it has the name of Pierre-Ernest Weiss (1865-1940) a French physicist who developed in 1907 the theory of ferromagnetism.

${ }^{21}$ See the patent US 4,467,696 granted to Ian McNab et al., in Aug. 28, 1984, for an "Electromagnetic projectile launcher with combination plasma/conductor armature", or the patent US 5,024,137 granted to Jon Schroeder in June 18, 1991, for an "Fuel assited electromagnetic launcher", etc.
} 
create cartridges based on FCG principle, while the projectiles could be the beneficiaries of the strong magnetic field created by the FCG-cartridge. An important aspect is also the portable power source: in this regard, we turned our attention to the new types of brushless homopolar generator.

\section{A possible power supply for modern electric weapons: the brushless homopolar generator with dielectric elements}

The homopolar generators began to be made with the experiment of 1831 made by Michael Faraday, who used a disk of conductive material under a perpendicular magnetic field; by means of some carbon brushes disposed on the disc spindle and on its periphery respectively, Faraday was able to obtain a potential and a fairly high current when turning the disk into a magnetic field. Such generators have since become known for their ability to generate strong currents and low voltages, which are appropriate for some industrial applications, especially for the electrolytic baths.

A particular embodiment of the homopolar generator is the use of a cylindrical or discoidal component made of dielectric material by applying a perpendicular magnetic field. When the magnetic field is applied and the above mentioned disk is rotated in the electric field, the capacitor charging occurs.

Although totally different from the classical scheme of the homopolar generator, this is also a form of application of the same Law of induction (stated by M. Faraday ${ }^{22}$ in 1831, and J. Henry ${ }^{23}$ in 1832) underlying the classic homopolar generator. Basically we are dealing with the formation of electrical charges within a rotor dielectric disc disposed in a perpendicular magnetic field. Although the physical phenomenon has long been known as such, the practical applications have been delayed.

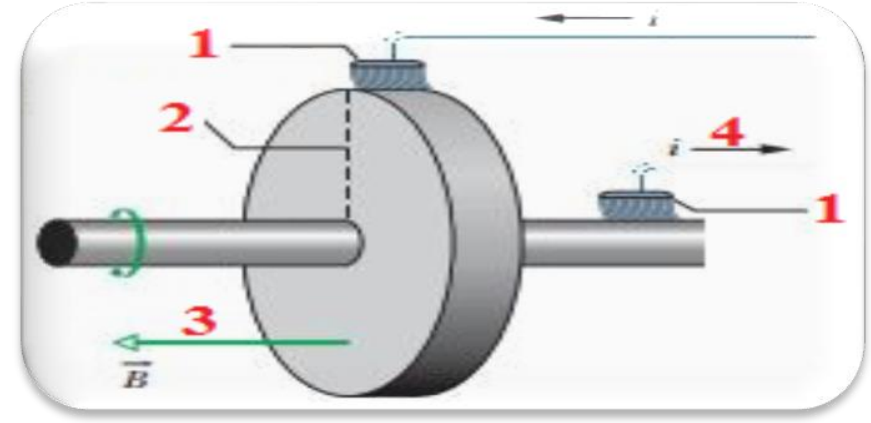

Fig. 5 Faraday generator operating principle: the collectors or brushes (1) take the current from the periphery of the rotor disk (2) and its shaft, both made of electroconductive material; in the situation where the rotor is subjected to the action of a perpendicular magnetic field (3). A strong current (4) is obtained.

\footnotetext{
${ }^{22}$ Michael Faraday (1791-1867), British scholar and physicist, he has major contributions to electromagnetism, optics and electrochemistry. Among his discoveries we can enumerate the electromagnetic induction, diamagnetism, electrolysis, and how the electromagnetic field interacts with light. In 1836 he invented the device called the "the Faraday Cage", designed to protect against the electromagnetic field and induction. At present, in the field of physics and chemistry, is used the so-called "constant Faraday", named in honor of physicist Michael Faraday, and represents the molar electric charge 96485,33289(59) C mol-1, where the elementary electric charge is $\mathrm{q} \approx 1,6021766 \times 10^{-19} \mathrm{C}$ and the number of Avogardo is $\mathrm{N}_{a} \approx 6,022141 \times 10^{-23} \mathrm{~mol}$. Also, in honor of Faraday the unit of measure of electrical capacity in International System was called "Farad".

${ }^{23}$ Joseph Henry (1797-1878), American scientist who served as the first secretary of the Smithsonian Institute. Among others, he stated the phenomenon of self-induction and mutual induction, working in parallel with Michael Faraday (1791-1867), but the latter was the first to highlight the phenomenon of induction and published the results of these observations.
} 
Among others, the subject of this paper is also to present the possibilities of military applications for this interesting type of brushless homopolar generator ${ }^{24}$. Virtually, a generator in the form of a homopolar electric capacitor, having a set of layered dielectric plates and conductive plates disposed in a magnetic field with at least one dielectric plate rotated relative to the rest of the plates, in order to generate electrical charges between the conductive plates.

A version of this generator has flat circular discs with a dielectric inner disk rotated relative to the other discs. The inner disk is placed between a pair of electrical conductive plates, which in turn are placed between two pairs of dielectric plates that are fixed to the conductive plates. A second embodiment uses concentric cylinders in a similar arrangement, the relative rotational motion of an inner dielectric being performed along a common axis for all cylindrical plates.

A brushless homopolar generator with dielectric ${ }^{25}$ material consists of a series of parallel capacitors connected and whose dielectric is in the form of a shaft driven by a motor. The capacitors are interconnected by positive and negative pairs. When the switch is in the open position, the homopolar generator consisting of a plurality of parallel capacitors produces and maintains electrical charges in its terminals. If there are used materials with suitable properties for capacitors, the electrical charge in the terminals can reach high values, corresponding to the dielectric characteristics, its velocity and the presence of the magnetic field.

The scheme of such a generator may contain a battery or a bank of batteries which, by means of a switch, powered the load circuit. This circuit can be essentially represented by the electric motors that drive the wheels of a vehicle, and the accumulators are the conventional power source for such an electrically driven vehicle. When the switch is closed, the batteries will power the engine.

In order to charge the batteries, the switch is periodically operated in the sense of closing the circuit formed by the battery and the terminals of the homopolar generator. In this way, the electrical charges obtained by operating the homopolar generator with its rotational dielectric material (driven by an engine) can be used to recharge the battery; In this way, the system will be able to function for a long time, its autonomy being therefore very high.
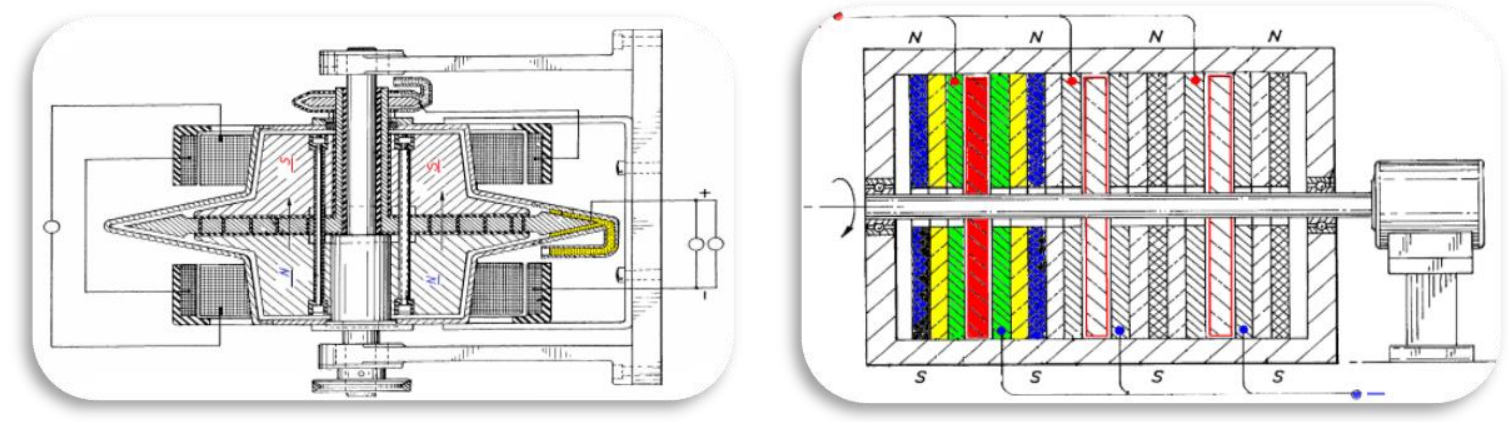

Fig. 6 On the left, the homopolar generator with liquid metal (mercury) collector invented by A. Sears ${ }^{26}$. On the right, the general scheme of a homopolar generator with dielectric materials and brushless operation: the generator package of plates is composed of a pair of three fixed plates (in total 6 plates) for each dielectric rotor, mounted to the dielectric shaft. In the drawing, the permanent magnet is depicted in blue, the stative dielectric material is yellow, the electrically conductive material is green and the dielectric rotor is red. It can be seen that the pairs of three fixed plates are axially disposed in front of and behind the dielectric rotor.

Such a homopolar generator with dielectric material is inserted into a housing, preferably made of polycarbonate plastic. An external motor is used to drive a rotary shaft made

\footnotetext{
${ }^{24}$ Lucian Ștefan Cozma, research report in Faculty of Physics, Măgurele, 2020.

${ }^{25}$ According the patent US 6,051,905 granted to Richard Clark in 2000.

${ }^{26}$ According the patent US 3,185,877 granted in May 25, 1965 for an "Direct current homopolar generator".
} 
of diamagnetic material and secured by a pair of bearings mounted at the ends of the housing. The capacitors are made of circular plates and use materials with good properties, such as those proposed by inventor E. Baldwin in U.S. Patent No. 4,870,538 ${ }^{27}$.

Within such a scheme, we have a plate containing a circular element composed of a permanent magnet, its magnetization being realized in a direction perpendicular to the central axis of the motor shaft made of diamagnetic material. By supporting the magnet and located between it and the electrode conductor there is a dielectric plate.

These plates are attached to the housing and separated from the shaft. The next element in the package of plates comprises a dielectric circular plate fixed to the shaft and rotating with it. The outer edge of the dielectric plate is spaced apart from the inner edge of the housing. The plates package is however completed with an electroconductive plate, a fixed dielectric plate and an external magnet. This whole package of plates made of various materials performs the homopolar generator circuit coupled to one of the capacitors.

But such a real generator may comprise a plurality of such packets, each corresponding to one capacitor. Thus, a magnet will correspond to a statoric dielectric plate, to an electrically conductive plate separate from the second electroconductive plate, to a dielectric plate, and another magnet along with the rotary dielectric. At an intermediate position there is a statoric dielectric plate, also a second electro-conductive plate and another statoric dielectric plate. The electrically conductive plates are connected to a terminal. An engine drives the shaft at speeds of 6,000-15,000 rpm. The offset electric motor voltage is given by the relation

$$
\mathrm{E}=\mathrm{vBk}
$$

where $\boldsymbol{B}$ is the magnetic flux $x^{28}$ of permanent magnets, the field being applied in a direction

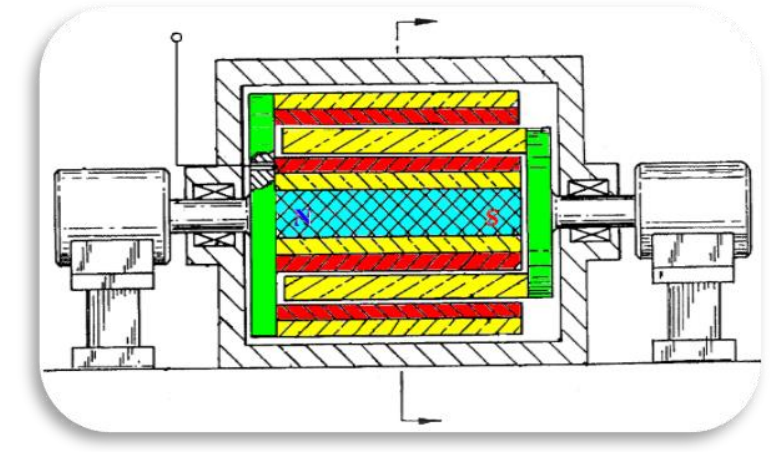

Fig. 7 The components of the homopolar generator with dielectric material are concentrically arranged and optionally driven by two motors simultaneously: in blue is depicted the axially polarized magnetic core, in yellow are illustrated the dielectric components, in red are depicted the components which are good conductors of electricity and in green are illustrated those insulator elements used to fasten all the other components. In fact, the generator can work when is driven by both engines (illustrated on the left and the right side of the generator) or only one of them.

parallel to the plane formed by the dielectric plates, $\boldsymbol{v}$ is the axis rotation speed and $\boldsymbol{k}$ is a constant related to the permittivity or the dielectric constant of the capacitor. This relation results in the energy dependence of the magnetic flux and the rotation speed. Preferably, the magnetic materials with high permeability (for magnetic discs) such as ceramic magnets or NDIE (Neodymium Iron Boron) are used in this case. Such device could be a compact source of high amperage used by the armatures of electric weapons, especially those in the railgun category.

\footnotetext{
${ }^{27}$ The patent US 4,870,538 granted to Ewart Baldwin in September 26, 1989 for an High energy density capacitor and method of fabrication.

${ }^{28}$ Although the notation $B$ typically refers to magnetic induction, the original text of the patent speaks about the "magnetic flux" not just about induction.
} 


\section{Conclusions}

Taking into account the above, we can assume that in the near future the technology of electric weapons could be improved in the following ways:

1. the use of active-passive electromagnetic ammunition, where the explosion of a small powder load produces (by applying electromagnetic pulse technology) an intense electromagnetic field designed to interact with the magnetic field induced by the magnetic rails; the projectile itself would be passive from the electromagnetic point of view;

2. the use of active electromagnetic ammunition, in which the projectile winding is powered by internal sources located either inside the projectile or in the cartridge;

3. the use of devices based on hybrid technologies, combining the organizational and operational schemes of classic (pyrotechnic) weapons and of electromagnetic weapons such as the coilgun/railgun, FCG (flux compression generator) or EMP (electromagnetic pulse weapons); by applying the Z-Pinch effect (the scheme of coaxial plasma detonator, also called the magnetic piston);

4. the use of Bitter-type electromagnetic accelerators and hybrid projectiles made of solid magnetic material and ionized gases;

5. the use of advanced, compact, economical power supplies such as brushless homopolar generators powered by improved models of thermal or electric motor.

Therefore, we expect the emergence in the near future of new and more powerful models of electric weapon, also more economical and reliable. That is expected to become an important episode in the generic process known as RMA (revolution in military affairs).

\section{Bibliography (Patents)}

(patent number, publication date, applicant, patent title)

US4694729, Sep 22, 1987, Rockwell International Corporation, Electromagnetic launcher assembly.

US4796511, Jan 10, 1989, Wisconsin Alumni Research Foundation, Electromagnetic projectile launching system. US4901621, Feb 20, 1990, Gt-Devices, Superconducting projectile for a rail gun and the combination of a rail gun with a superconducting projectile.

US5183957, Feb 2, 1993, The University Of Texas, System Method and construction for control of current distribution in railgun armatures.

US5191164, Mar 2, 1993, USA Department Of Energy, Hybrid armature projectile.

US5233903, Aug 10, 1993, Israel- Atomic Energy Commission, Soreq Nuclear Research Center, Gun with combined operation by chemical propellant and plasma.

US5483863, Jan 16, 1996, Dyuar Incorporated, Electromagnetic launcher with advanced rail and barrel design.

US8701639, Apr 22, 2014, George Arthur Proulx, Open railgun with steel barrel sections.

US20100194212, Aug 5, 2010, George Arthur Proulx, Railgun system.

US8302584, Nov 6, 2012, Weimin Lu, Rail gun launcher.

US8322328, Dec 4, 2012, Lawrence Livermore National Security, Llc Solid-to-hybrid transitioning armature railgun with non-conforming-to-prejudice bore profile.

US20120000450, Jan 5, 2012, Lawrence Livermore National Security, Llc Solid-to-Hybrid Transitioning Armature Railgun With Non-Conforming-To-Prejudice Bore Profile.

US8590453, Nov 26, 2013, USA- The Secretary Of The Army, Extending boom for stabilizing projectiles launched from an apparatus.

US4765222, Aug 23, 1988, The Boeing Company, Electrostatic kinetic energy weapon.

US4791850, Dec 20, 1988, Minovitch Michael Andrew, Electromagnetic launching system for long-range guided munitions.

US4796511, Jan 10, 1989,Wisconsin Alumni Research Foundation, Electromagnetic projectile launching system. US4867036, Sep 19, 1989, Ford Aerospace \& Communications Corporation, Electromagnetic gun bore rider.

US5127308, Jul 7, 1992, The Boeing Company, Distributed energy store electromagnetic railgun.

US5275083, Jan 4, 1994, The United States Of America As Represented By The United States Department Of Energy, Skirted projectiles for railguns. 
US5417140, May 23, 1995, Mitsubishi Jukogyo Kabushiki Kaisha, Flying object acceleration method by means of a rail-gun type two-stage accelerating apparatus.

US6142131, Nov 7, 2000, The United States Of America As Represented By The Secretary Of The Army, Electromagnetic launcher with pulse-shaping armature and divided rails.

US6965215, Nov 15, 2005, General Atomics, Capacitor pulse forming network with multiple pulse inductors. US7077047, Jul 18, 2006, Joseph Franklin Frasca, Electromagnetic propulsion devices.

US7503249, Mar 17, 2009, General Atomics, Barrels for electromagnetic guns.

US7514820, Apr 7, 2009, General Atomics, Capacitor pulse forming network with multiple pulse inductors.

US7724401, May 25, 2010, Kung-Cho Sheng, Image scanner having wireless carriage module.

US8302584Nov 6, 2012, Weimin Lu, Rail gun launcher.

US8677878, Mar 25, 2014, Lockheed Martin Corporation, Thermal management of a propulsion circuit in an

US8714991, May 6, 2014,Rittal Gmbh \& Co. Kg, Busbar adapter comprising a mounting rail for attaching a switching device.

US9476668, Oct 25, 2016, Enig Associates Inc., Hypervelocity projectile launching system.

US9551548, Jan 24, 2017, Borja SIERRA IZQUIERDO, Barrel and an electromagnetic projectile launching system.

US20050016373, Jan 27, 2005, Macdougall Frederick W., Method for shipboard operation of electromagnetic gun and rotating pulse forming network.

US20050126381, Jun 16, 2005, Macdougall Frederick W.,Rotating pulse forming network for shipboard operation of electromagnetic gun and capacitor module.

US20050155487, Jul 21, 2005, Frasca Joseph F., Improvements to Electromagnetic Propulsion Devices.

US20050168195, Aug 4, 2005, General Atomics, Capacitor pulse forming network with multiple pulse inductors. US20060001916, Jan 5, 2006, Primax Electronics Ltd., Image scanner having wireless carriage module.

US20060243124, Nov 2, 2006, General Atomics, Barrels for electromagnetic guns

US20060290399, Dec 28, 2006, General Atomics, Capacitor Pulse Forming Network with Multiple Pulse Inductors.

US20070277668, Dec 6, 2007, Frasca Joseph F, Two Rail Electromagnetic Gun.

US20130189862, Jul 25, 2013, Rittal Gmbh \& Co., Kg, Busbar adapter comprising a mounting rail for attaching a switching device.

US20150091478, Apr 2, 2015, Velocity Magnetics, Inc., Solid state energy storage and management system. 